\title{
Mechanical behavior and failure mechanisms of Li-ion battery separators
}

Sergiy Kalnaus ${ }^{*}$, Yanli Wang, John A. Turner

Oak Ridge National Laboratory, Oak Ridge, TN 37831-6164, USA

\begin{abstract}
Anisotropic mechanical properties were experimentally determined and compared for three types of commercially available Li-ion battery separators: Celgard 2325, Celgard PP2075 dryprocessed polymer separators, and DreamWeaver Gold 40 non-woven separator. Significant amount of anisotropy of properties was determined, with the Young's modulus being different by up to a factor of 5 and ultimate strength being different by a factor of 10 between orthogonal directions within a polymer separator layer. Strain rate sensitivity was investigated by applying strain rates ranging from $1 \cdot 10^{-4} \mathrm{~s}^{-1}$ to $0.1 \mathrm{~s}^{-1}$. Significant strengthening was observed and the strain rate strengthening coefficients were determined for both elastic modulus and yield stress in case of polymer separators. Digital image correlation technique was used to measure and map the strains over the specimen's gage section. Significant strain concentration in bands running perpendicular to the tensile axis was observed in polymer separator samples oriented in transverse direction. Such localized necking allows for extremely high strains close to $300 \%$ to develop in the material. The failure mode was remarkably different for all three types of separators which adds additional variable in safe design of Li-ion batteries for prevention of internal short circuits.
\end{abstract}

Keywords: Lithium-ion; separator; polymer; rate sensitivity; mechanical; safety

Notice: This manuscript has been authored by UT-Battelle, LLC under Contract No. DE-AC0500OR22725 with the U.S. Department of Energy. The United States Government retains and the publisher, by accepting the article for publication, acknowledges that the United States Government retains a non-exclusive, paid-up, irrevocable, world-wide license to publish or reproduce the published form of this manuscript, or allow others to do so, for United States Government purposes. The Department of Energy will provide public access to these results of federally sponsored research in accordance with the DOE Public Access Plan (http://energy.gov/downloads/doe-public-access-plan).

\footnotetext{
* Corresponding author

E-mail address: kalnauss@ornl.gov (S. Kalnaus)
} 


\section{Introduction}

Successful electrification of automotive drivetrains depends in large part on improvement of safety of on-board energy storage systems, which nowadays are almost exclusively represented by lithium-ion secondary batteries assembled in packs. These systems store significant amount of energy and the rapid release of it, such as in the event of a short circuit, can lead to catastrophic events such as thermal runaway. In a liquid electrolyte-based Li-ion cell of any shape factor the charge is transported between the electrodes by ions, while the electrons carry the charge through the electrodes, metal current collectors, and to the external circuit. The component that prevents the electronic current occurring between the electrodes, i.e. prevents the direct contact between them, is termed a separator. This component thus should address the following requirements: a) it should be porous to allow liquid electrolyte to carry lithium ions, and b) it should have sufficient strength to prevent contact between the electrodes. The latter is typically assessed by a puncture strength parameter. There are also additional requirements such as low thermal shrinkage.

The second requirement (mechanical strength) is the primary subject of the present study. Currently two major measures are used to address this: - Young's modulus in machine direction (MD) and puncture strength [1]. Machine direction is chosen for dry processed separators since it is the direction of windings during jellyroll processing and thus the material should withstand tension from winding machine. The puncture test follows the ASTM D4830 standard [2] which describes puncture strength as a maximum load applied to a needle with $1 \mathrm{~mm}$ tip radius at a penetration rate of $0.5 \mathrm{~mm} / \mathrm{s}$. The current requirements are $100 \mathrm{MPa}$ for the Young's modulus and $300 \mathrm{~g}$ for puncture strength [3].

While the above parameters may be sufficient to address integrity of separators during battery manufacturing and transport, they do not provide a full understanding of mechanical behavior of these membranes. For instance, as demonstrated in the current report, the elastic modulus of polymeric separators appears to be a function of loading rate, which is not reflected in a $100 \mathrm{MPa}$ requirement. Better understanding of mechanical properties of separators is especially relevant when the behavior of the battery under external mechanical loading (such as in the event of crush or drop) is considered. Constitutive models describing mechanical behavior of separator can be used in numerical models for better predictions of battery response and improvements in safety $[4,5]$. In addition, during battery charging and discharging, separator can deform due to swelling of electrodes and thus change the effective properties in terms of ionic conductivity [6].

There are number of studies on Li-ion battery separators addressing the mechanics that have been published relatively recently [7-12]. The early study [7] first reported changes in porosity due to mechanical load and investigated the separator safety shutdown mechanism as a function of temperature. In other reports, tensile and compressive experiments on commercial separators have been discussed [8-12]. Such experiments usually involve a single type of separator, often extracted from a commercial Li-ion cell after its disassembly $[4,5]$ and thus providing limited 
knowledge of the separator type, manufacturer, and its composition. Most studies involve polymer separators, for example Celgard 3501 in [12] or Celgard C480 in [9]. Both dry and wet experiments in compression were performed in [12] and the poroelastic model was subsequently proposed in [13] to describe the effect of liquid filling the separator pores under compression. The probably most comprehensive study is reported in [11] where separators from three manufacturers were tested for crack propagation under tension in different directions with in-situ atomic force microscopy (AFM). Finally, thermomechanical behavior of several polymer separators was studied in [10].

The current investigation expands the existing knowledge of mechanical behavior of porous membranes used as Li-ion battery separators by i) comparing the behavior of three different materials (viz. Celgard 2325, Celgard PP2075, and DreamWeaver Gold 40); ii) investigating anisotropy and strain rate sensitivity; iii) investigating inhomogeneity of strain distribution under tensile loading and fracture mode. For that purpose, two porous polymer membranes manufactured by Celgard $\AA$ and one nonwoven separator manufactured by Dream Weaver Inc were tested under wide range of strain rates. Digital image correlation (DIC) technique was used to observe strain distribution in samples as the test progressed. In addition to reporting basic mechanical properties of the materials, such as elastic modulus and yield stress, the paper discusses significant differences in strength, strain distribution, and failure mode among the three materials, which may provide guidance for addressing the safety of Li-ion batteries.

\section{Experiments}

MTS load frame with $50 \mathrm{lbs}(\sim 0.2 \mathrm{kN})$ load cell with $0.1 \mathrm{~N}$ measurement accuracy was used for the experiments. Wedge grips were used to hold the sample; rubber inserts were placed inside the grips to prevent tearing of separator (Fig. 1). Samples were $8 \mathrm{~cm}$ long strips with width equal to $2 \mathrm{~cm}$. The gage length was kept at $40 \mathrm{~mm}$. Samples were cut from the as received sheets of separator material using razor blade. Tests were done under displacement control, dry conditions. Strain rates ranging from $1 \cdot 10^{-4} \mathrm{~s}^{-1}$ to $0.1 \mathrm{~s}^{-1}$ were applied. Based on the reports on the measurements of Li-ion pouch cell swelling during electrochemical cycling, the lower strain rate may be considered as corresponding to $1 \mathrm{C}$ cycling of a $\mathrm{LiCoO}_{2}$ vs Graphite pouch Li-ion cell [12]. Here the term C-rate is used and $\mathrm{C}$ indicates the time required to completely charge or discharge the battery, i.e. $1 \mathrm{C}$ represents charge in 1 hour and $0.5 \mathrm{C}$ would correspond to charge in 2 hours. Digital image correlation was used to measure the strain distribution. The speckle pattern for DIC was made manually by using a permanent marker. The decision was made to use this method rather than commonly used spray painting technique due to possible alteration of properties of separator by paint or delamination of paint during the test. VIC 2D software was used for image analysis and calculation of strains. A small tensile load of approximately $0.1 \mathrm{~N}$ was applied prior to test to keep the sample straight and avoid any droop. The reference image for DIC was recorded in this initial state. Microstructure of separators was observed using Hitachi S-4800 field emission scanning electron microscope (FE-SEM) using $5 \mathrm{kV}$ accelerating voltage. A thin layer of gold was sputtered on the samples to avoid charge build up in SEM. 
Three types of separator were tested: Celgard 2325, Celgard PP2075, and DreamWeaver Inc 40 um thick microfiber-based separator. Their description is arranged in Table 1 (porosity and thickness was determined by the corresponding manufacturer). Commercial films were obtained from Celgard LLC and Dreamweaver International and were used in as-received condition. Two Celgard ${ }^{\circledR}$ separators were chosen to study the difference between triple-layer and single-layer polymer membranes. While 2325 separator has three layers of Polypropylene/Polyethylene/Polypropylene (PP/PE/PP), the PP2075 is a high-porosity single PP layer, developed by Celgard for high-rate applications. The DreamWeaver Gold40 is a nonwoven sheet made of Kevlar-type (para-aramid) fibers with very high porosity (Table 1). All of the experiments were conducted at room temperature in ambient atmosphere. Tensile behavior was determined in two orthogonal directions, as shown in Fig. 2 (b). It is expected that there is a certain degree of anisotropy due to the separator manufacturing procedure and the properties in machine direction (MD) differ from those in transverse direction (TD).

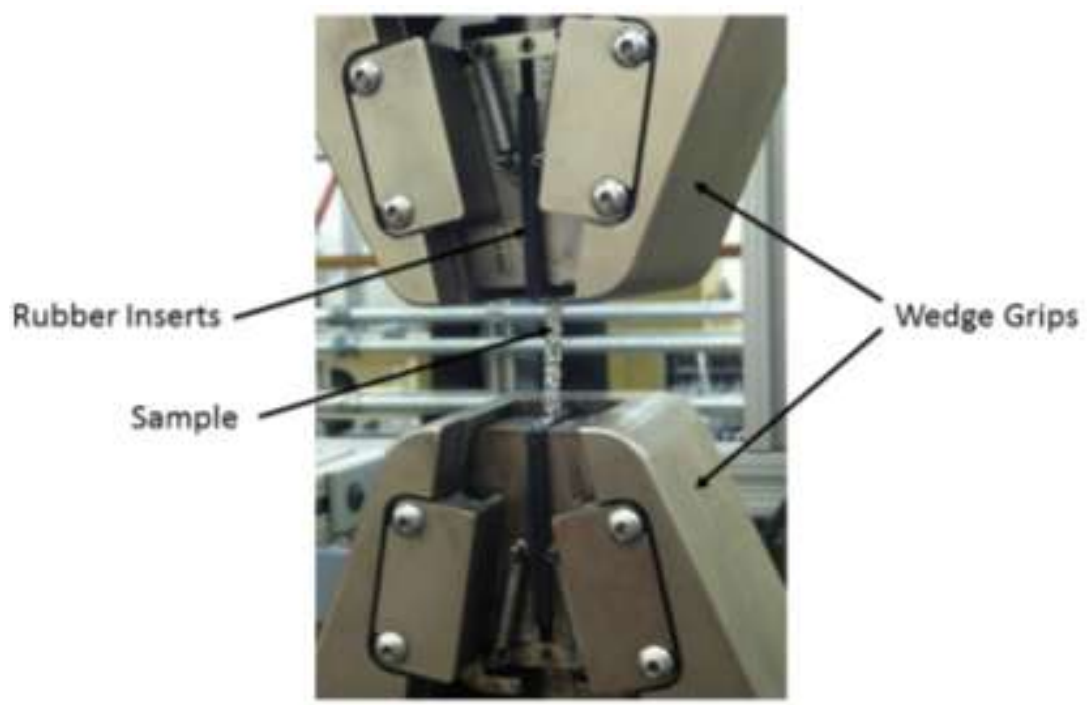

Fig. 1. Setup for separator tensile test.

Table 1. Separators nomenclature and description

\begin{tabular}{llll}
\hline Separator & Composition & $\begin{array}{l}\text { Thickness } \\
\boldsymbol{\mu m}\end{array}$ & $\begin{array}{l}\text { Porosity } \\
\mathbf{\%}\end{array}$ \\
\hline Celgard 2325 & PP/PE/PP & 25 & 39 \\
Celgard PP2075 & PP & 20 & 48 \\
$\begin{array}{l}\text { DreamWeaver Gold } \\
40\end{array}$ & $\begin{array}{l}\text { Non-woven para- } \\
\text { aramid fibers }\end{array}$ & 40 & 68 \\
\hline
\end{tabular}




\section{Results and discussion}

Comparison of tensile strength of the three separators is provided in Fig. 2(a). The results shown in Fig. 2(a) correspond to $1 \cdot 10^{-3} \mathrm{~s}^{-1}$ strain rate. Significant difference in behavior can be observed with the polymer separators displaying high anisotropy while the fiber-based DreamWeaver behaves isotropically. It is interesting to note that tensile strength of a single-layer Celgard PP2075 is higher than that of the triple-layer Celgard 2325. Overall, the ratio of the tensile strength in MD over tensile strength of TD is close to a factor of ten for Celgard 2325 and is higher for Celgard PP2075. This ratio remained constant for all applied strain rates used in the experiments. Such anisotropic behavior is consistent with expectations for dry processed polymer separators for batteries [8]. The anisotropy of mechanical properties is expected from the examination of microstructure, which is shown (Celgard 2325) together with designation of machine and transverse directions in Fig. 2(b). The microstructure consists of thicker crystallite lamellae running perpendicular to the machine direction connected via thin fibrils formed by dry crazing process.

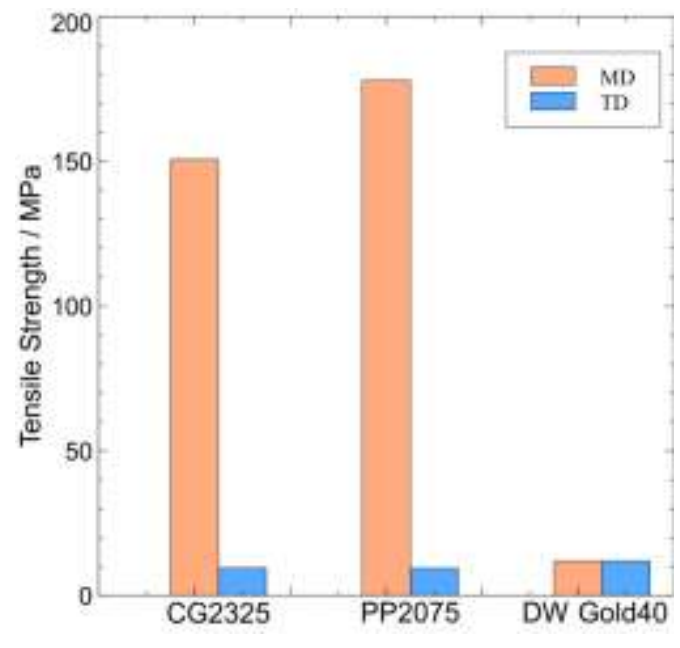

(a)

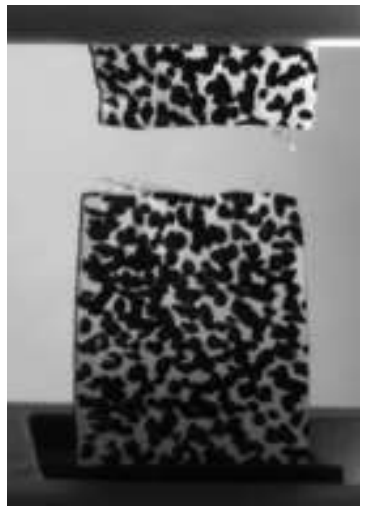

(c)

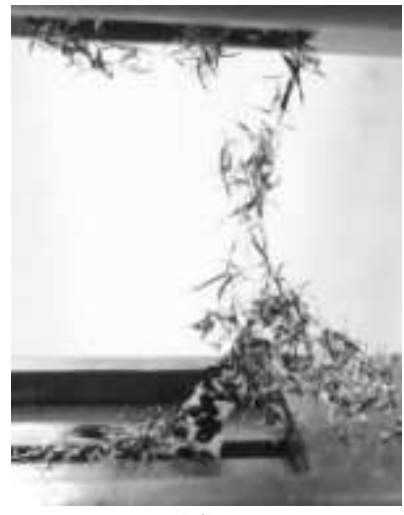

(d)

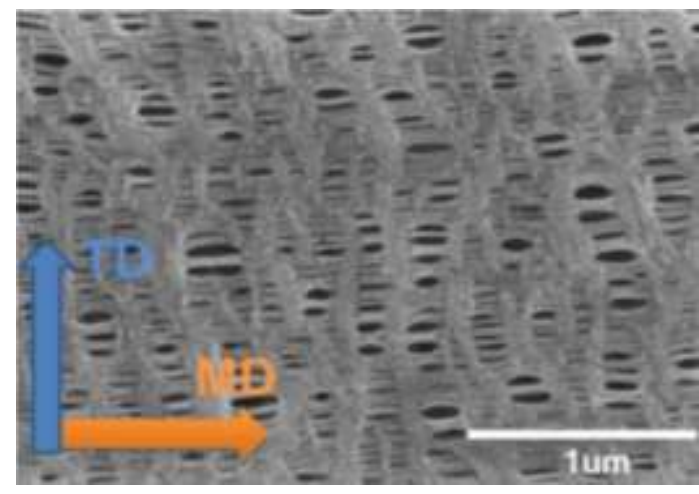

(b)

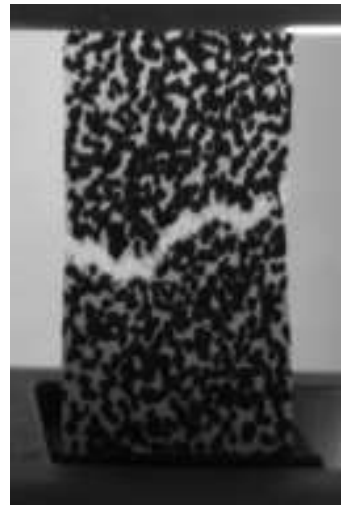

(e)

Fig. 2. Overall comparison of tensile strength and failure features. (a) comparison of tensile strength in orthogonal directions (CG stands for Celgard and DW is short for Dream Weaver); 
(b) designation of machine and transverse directions; (c) rupture of Celgard 2325; (d) rupture of Celgard PP2075; (e) rupture of DreamWeaver Gold 40.

One observation that clearly distinguishes two Celgard polymer separators needs to be mentioned. While the tensile failure of 2325 in machine direction proceeds as a straight tear perpendicular to the loading axis (Fig. 2(c)), the 2075 separator fails by splitting into multiple fibers (Fig. 2(d)). Such disintegration happens instantaneously as the separator shreds into thin strips. There is no observable necking preceding such abrupt failure. The strips are approximately $50-100 \mu \mathrm{m}$ wide and show rather sharp edges evident of tearing (Fig. 6(b)).

The tensile stress-strain curves of Celgard 2325 separator in transverse direction are shown in Fig. 3(a). The curves are plotted using engineering stress and strain. It is noted that conversion to true stress and strain variables rigorously speaking would not be valid, since the assumption of constant volume during the deformation no longer holds in case of deformation via crazing, as discussed further in this section. Very high strains were developed in TD samples, up to $300 \%$. Some tests in TD were not terminated with specimen rupture; rather either the boundaries of field of view of the DIC camera were exceeded as the specimen was pulled or the displacement limit of the crosshead was reached which stopped the test. It should be mentioned that it is hard to achieve replication of the breaking tensile strain due to high sensitivity of the material to the flaws which vary from sample to sample.

Some softening can be observed in TD oriented samples, where a clear peak stress is followed by a quasi-plateau (cold drawing) region where material flows with very high plastic strains. It should be mentioned that these high strains are localized within few bands that run across the sample perpendicular to the loading axis. The strain distribution (Fig. 3(a)) obtained via DIC demonstrates the formation of such bands in a TD loaded Celgard 2325 under $\dot{\varepsilon}=5 \cdot 10^{-3} \mathrm{~s}^{-1}$. The strain shown is $\varepsilon_{22}$, i.e. normal strain in the direction of tension; however localization is so pronounced that Von Mises strain obviously shows similar behavior. It can be seen from the strain map in Fig. 3, that the bands can accommodate strain which is several times higher in magnitude than the matrix strain. This scenario was observed under all of the applied strain rates. The micro-crazes, developed perpendicular to the loading axis, coalesce to form macroscopically observable bands accumulating major part of the strain. On the macro-level this is manifested by the formation of opaque and semi-transparent regions of the separator under tension. Such crazing a known mode of deformation for semi-crystalline polypropylene [14, 15] and occurs perpendicular to the principal tensile stress. Crazing is associated with formation of voids in the material thus violating the assumption of constant volume during deformation. Further examination of the TD-deformed separator reveals distorted microstructure with stretched fragments of lamellae rows (Fig. 3(b)) as well as formation of cracks in crystalline lamellae (Fig. 3(c)). Significant opening of pores can also be observed. 


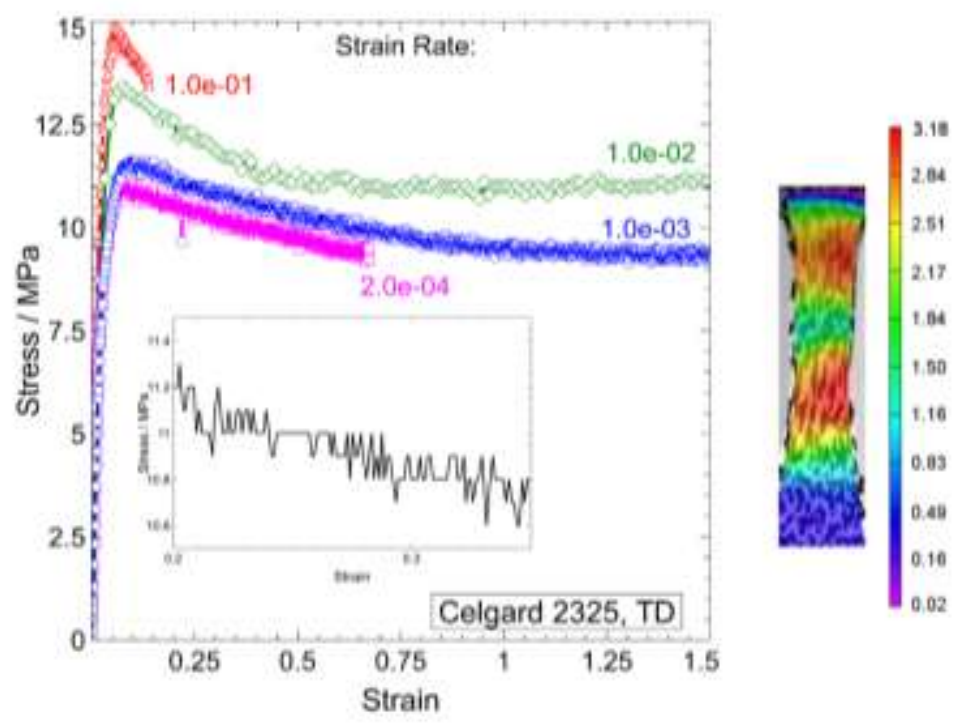

(a)

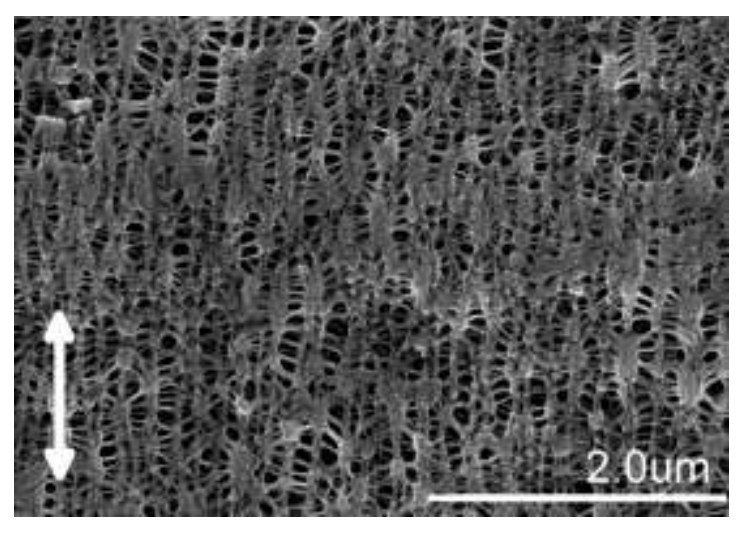

(b)

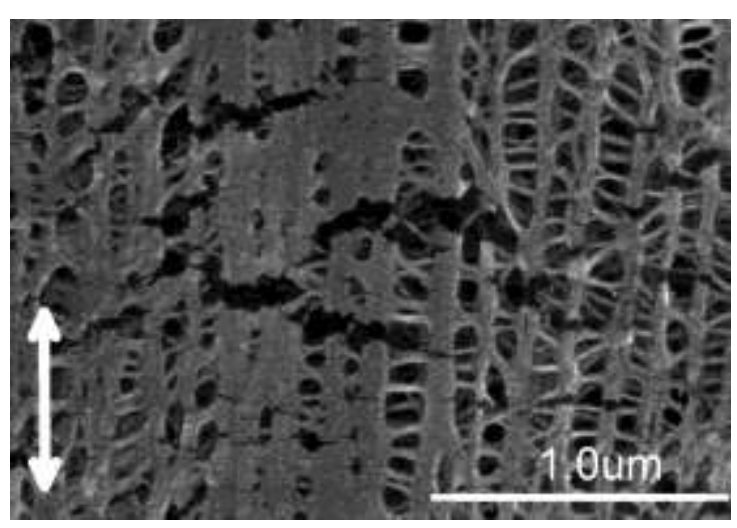

(c)

Fig. 3. Tensile behavior of Celgard 2325 in transverse direction. a)Stress-strain curves and the $\varepsilon_{22}$ strain distribution showing accumulation of strain in bands; b) SEM image of distorted microstructure after TD tension; c) High magnification SEM image showing cracks developed in lamellae under TD tension(the double-sided arrow indicates loading direction);

Similar behavior was observed in TD oriented Celgard PP2075 separator for which the tensile curves and a representative strain map are shown in Fig. 4. It should be mentioned however that the material did not achieve strain levels as high as a triple-layer PP/PE/PP 2325 separator. Nevertheless, strain localization in bands can be clearly seen although the difference between the normal strain within a band and matrix strain is not as pronounced. Such behavior was observed in all of the cases regardless of the applied strain rate. Unlike the triple-layer membrane, the single-layer PP separator does not display a peak yield stress in tension and there is no observable softening or hardening (Fig. 4); plateau stress remains constant until failure. 


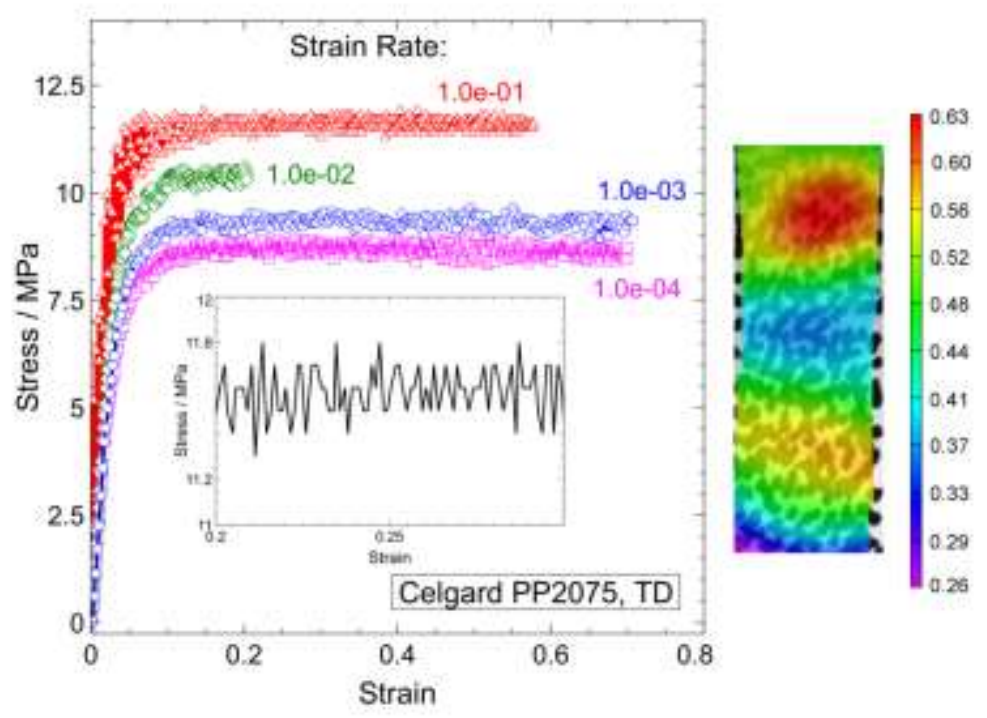

Fig. 4. Stress-strain curves of Celgard PP2075 in transverse direction. The $\varepsilon_{22}$ strain distribution shows accumulation of strain in bands.

Noticeable oscillations of tensile stress are present on the stress-strain curve following the yield point of Celgard 2325 and 2075 polymer membranes. These oscillations can be clearly seen in the inserts in Fig. 3(a) and Fig. 4 where parts of stress-strain curves are magnified to better illustrate the phenomenon. Amplitude of such jumps in stress appears to be higher in case of single-layer 2075 separator. For this separator, the magnitude of the stress oscillations is also a function of applied strain rate and is apparently higher with higher rate of specimen extension (Fig. 4). Based on the available research on the above phenomenon [16-20], the most common rationale behind necking stress oscillations has been proposed as a release of heat via a two-stage process. In the first stage crystallization of the polymer causes strain hardening and thus increases stress. This is followed by release of crystallization heat and drop in stress [16]. It should be mentioned that heat generation has been observed associated with polymer drawing $[18,19]$, and the thermo-mechanical model describing the process has been evaluated [18]. At the same time, the X-ray microdiffraction experiments did not observe any significant difference in degree of crystallinity among the bands developed as a result of drawing in syndiotactic polypropylene [20] and therefore the stress oscillations might not be the result of crystallization process.

Tension in machine direction results in a strikingly different behavior for the two polymer separators under investigation. As was mentioned above, there is an order of magnitude difference in tensile strength (Fig. 2(a)) distinguishing the two orthogonal directions. This can be explained by higher tensile strength of fibrils produced via periodic crazing during the separator manufacturing. Such pre-deformation during processing is analogous to strain hardening in metals and increases the tensile strength by orienting polymer chains. 
The stress-strain response in MD is shown in Fig. 5 for Celgard 2325 and in Fig. 6 for Celgard PP2075. In both cases homogenous distribution of tensile strain can be observed from the DIC strain maps. Little variations (less than $1 \%$ difference across the sample), comparable to errors of image processing can be detected; however there is no evidence of large strain accumulation in bands leading to micro-necking, characteristic of tension in TD. Strain distribution remains uniform until failure, which in case of PP2075 occurs by disintegration into multiple small pieces (Fig. 2(d), Fig. 6(b)).

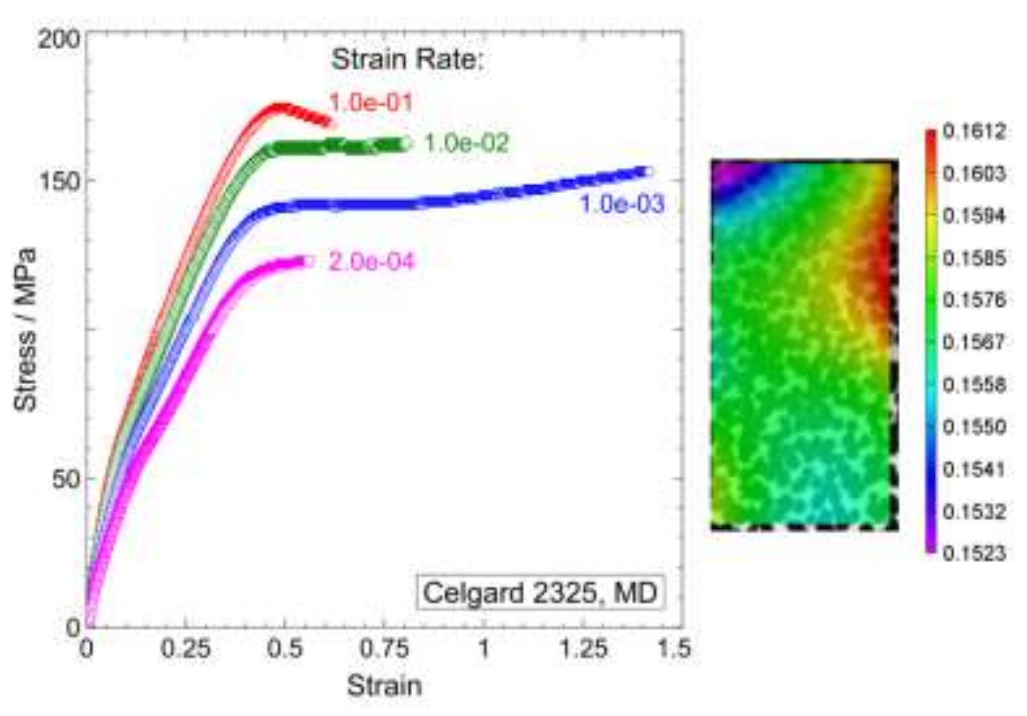

(a)

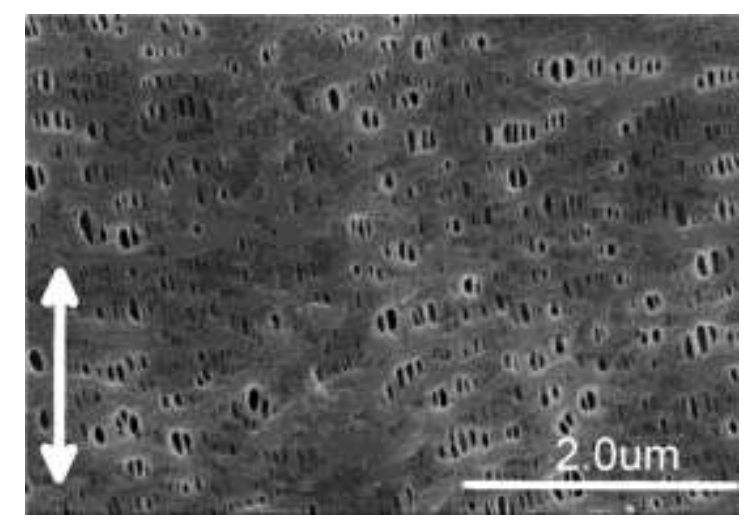

(b)

Fig. 5.Tensile behavior of Celgard 2325 in machine direction. a) Stress-strain curves and representative $\varepsilon_{22}$ strain distribution; b) SEM showing lamellae widening after tension in MD (double-sided arrow indicates the loading direction). 


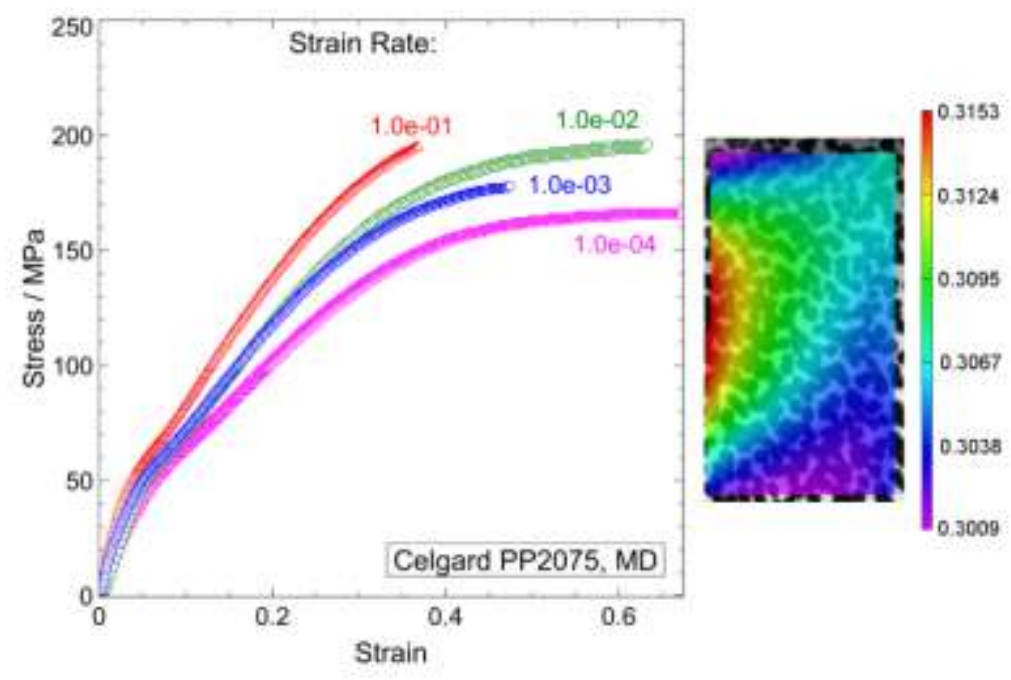

(a)

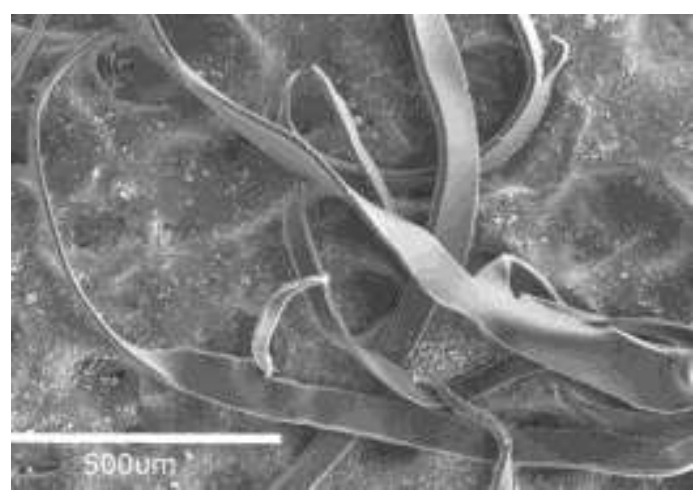

(b)

Fig. 6. Behavior of Celgard PP2075 in machine direction: a) Stress-strain curves and representative $\varepsilon_{22}$ strain distribution; b) Strips of material observed during tensile failure.

When stretched in machine direction, the load is mostly sustained by the fibrils that run parallel to the loading direction, - these fibrils have been already work hardened during the separator manufacturing. The microstructure of Celgard 2325 after tension in machine direction (Fig. 5(b)) is thus similar to that of un-deformed separator; however widening of the lamellae under tension can be observed. With extended deformation this widening may lead to separation of lamellae with formation of new fibrils.

It should be noted, that when tested under $\dot{\varepsilon}=1 \cdot 10^{-3} \mathrm{~s}^{-1}$ the Celgard 2325 separator displayed significant strain hardening (Fig. 5). Figure 5 shows a noticeable amount of work hardening that follows the constant stress plateau of the stress-strain curve in tension. This result appears to be repeatable (all three samples tested under this strain rate displayed this behavior). Such work hardening is in part expected due to deformation mechanism via crazing, which explains original higher strength in MD compared to TD. It is interesting to note however that 
such work hardening was only present in triple-layer separators and was observed under only one strain rate. In addition, no stress oscillations were observed in tensile stress-strain curves in manufacturing direction despite an obvious presence of cold drawing. Further research is needed to address this phenomenon in more detail.

Figures $3-6$ clearly show strain rate sensitivity in both polymeric Celgard separators. In order to quantify this effect, the tensile elastic modulus and yield was determined from the stressstrain curves in both orthogonal directions at different strain rates. It should be mentioned that MD stress-strain curves display several linear regions with different slopes separated by bends, or "knees" (Figs. 5, 6) that can be mistaken for yield points. In order to make sure the elastic regime is measured, the modulus is determined from the portion of the curve corresponding to the small strains not exceeding $1.5 \%$. This rule was used for processing data in both machine and transverse directions; however the linear region of the stress-strain curves in TD is clearly defined.

Determination of the yield stress in machine direction is rather ambiguous since there are several points along the stress-strain curve when a "knee" can be observed and a first nonlinearity occurs at a rather low strain (close to 1\%). This is especially the case for the Celgard PP2075 single-layer membrane where a yield point cannot be clearly defined. For the Celgard separators loaded in MD, the yield stress was defined as the maximum stress before the plateau (cold drawing) region. While this definition is not necessarily strict it allows comparison with the yield stress in TD, where a highly pronounced sharp yield point is present.

Figure 7 shows change in Young's modulus and yield stress in TD as a function of applied strain rate for both materials. The strain rate dependence can be described as [21,22]

$$
\left\{\begin{array}{l}
E=E_{0}\left(1+s_{1} \ln \left(\frac{\dot{\varepsilon}}{\dot{\varepsilon}_{0}}\right)\right) \\
\sigma_{y}=\sigma_{y}^{0}\left(1+s_{2} \ln \left(\frac{\dot{\varepsilon}}{\dot{\varepsilon}_{0}}\right)\right)
\end{array}\right.
$$

with the strain rate strengthening coefficient $s_{1}$ and $s_{2}$ easily determined from Fig. 7 as $s_{1,2}=\partial\left(E, \sigma_{y}\right) / \partial \ln \dot{\varepsilon}$ and $\dot{\varepsilon}_{0}=1 \cdot 10^{-4} \mathrm{~s}^{-1}$. The corresponding parameters describing strain rate sensitivity for both Celgard 2325 and Celgard PP2075 are arranged in Table 2. 


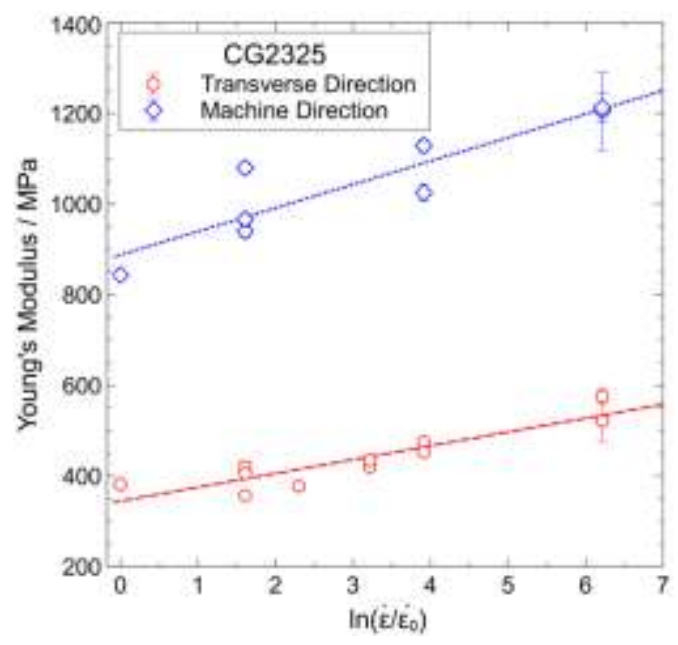

(a)

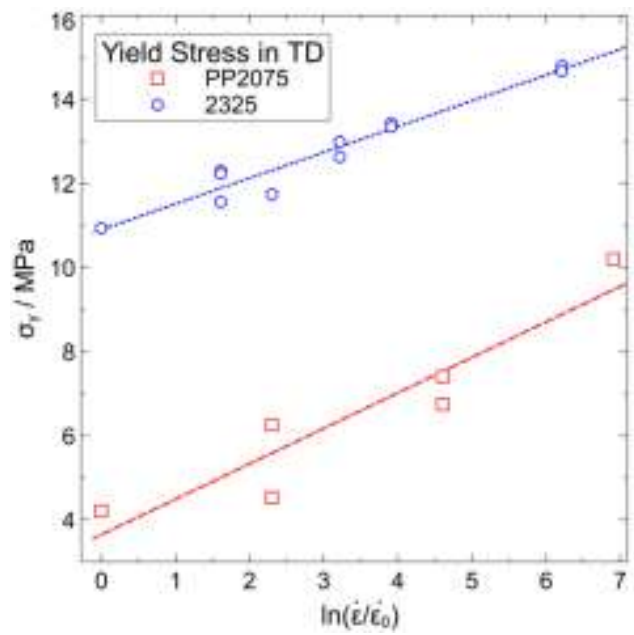

(c)

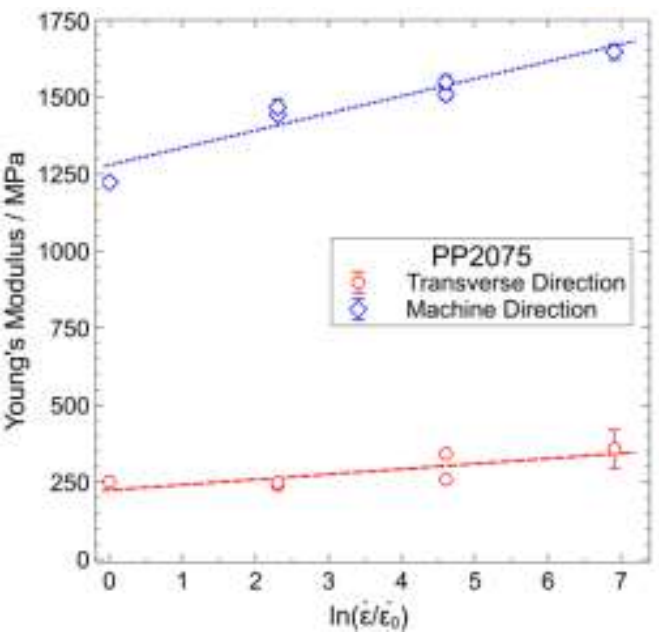

(b)

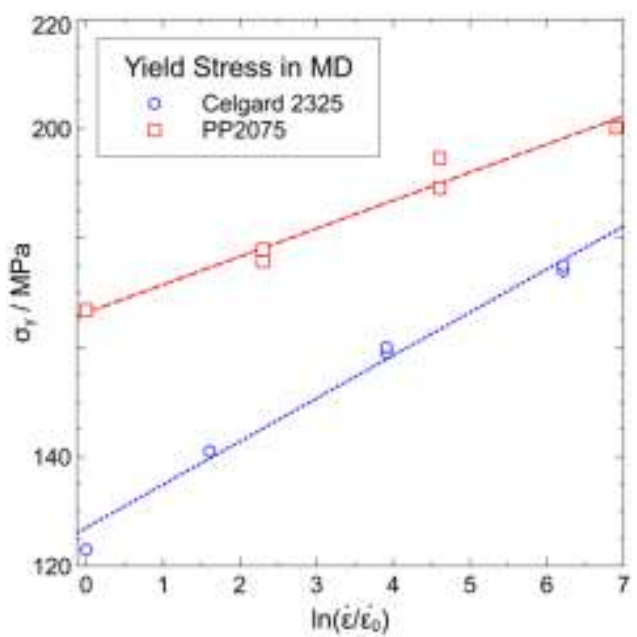

(d)

Fig. 7. Strain rate sensitivity of elastic modulus of a) Celgard 2325 and b) Celgard PP2075; c) strain rate sensitivity of yield stress in transverse direction; d) strain rate sensitivity of yield stress in machine direction.

Table 2. Strain rate strengthening coefficients of Celgard separators in two directions

\begin{tabular}{|c|c|c|c|c|c|c|c|c|}
\hline \multirow[b]{2}{*}{$\begin{array}{c}\text { Celgard } \\
\text { Separator }\end{array}$} & \multicolumn{4}{|c|}{ TD } & \multicolumn{3}{|c|}{ MD } & \\
\hline & $\begin{array}{c}E_{0}, \\
\mathrm{MPa}\end{array}$ & $s_{1}$ & $\begin{array}{c}\sigma_{y}^{0} \\
\mathrm{MPa}\end{array}$ & $s_{2}$ & $\begin{array}{c}E_{0}, \\
\mathrm{MPa}\end{array}$ & $s_{1}$ & $\begin{array}{c}\sigma_{y}^{0} \\
\mathrm{MPa}\end{array}$ & $s_{2}$ \\
\hline 2325 & 344.4 & $8.8510^{-2}$ & 10.9 & $5.6210^{-2}$ & 887.6 & $5.8410^{-3}$ & 127.0 & $6.210^{-2}$ \\
\hline PP2075 & 224.4 & $7.6110^{-2}$ & 3.65 & 0.23 & 1279.1 & $4.3810^{-3}$ & 166.3 & $3.110^{-2}$ \\
\hline
\end{tabular}


The third type of separator investigated in the current report was the DreamWeaver Gold 40, which is a highly porous ( $70 \%$ porosity) micro-fiber based membrane (Table 1) that was developed to minimize thermal shrinkage and provide high porosity for better transport through electrolyte. This is apparently achieved through combination of large and small fibers in the microstructure with large fibers providing load bearing capability and small fibers network resulting in high porosity. This microstructure illustrating this approach is shown in Fig. 8 (a).

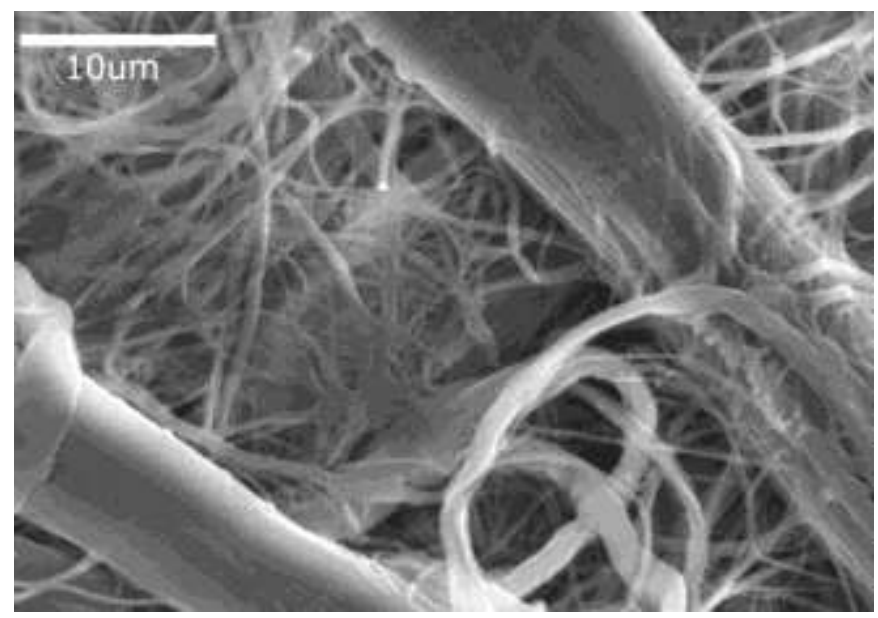

(a)

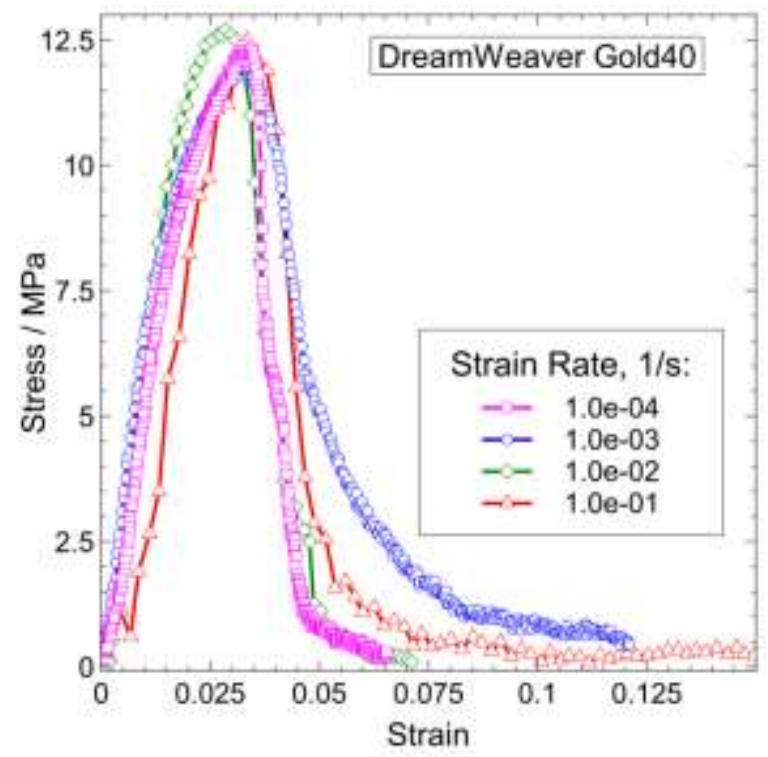

(b)

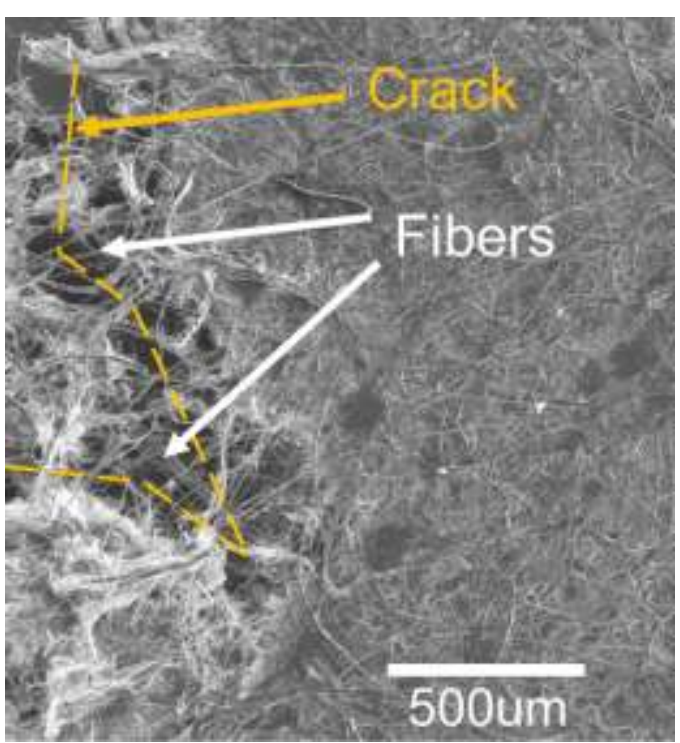

(c)

Fig. 8. Tensile behavior of DreamWeaver Gold 40 separator: a) Detail of microstructure; b) Stress-strain curves; c) Failure mode with fiber pullout.

Tensile strength of this separator appears to be rather low (Fig. 8 (b)) and is very similar to that of Celgard separators when tested in transverse direction (Fig. 2 (a)). The fracture strain 
however is only $3 \%$, - up to 100 times less than that of Celgard polymer separators. The tensile properties were obviously isotropic and there was no strain rate effect. It is interesting to note that the separator does not break apart immediately upon reaching the critical load, but is capable of still sustaining some load via fibers pulled out from the mat (Fig. 8 (c)). Thus the load decays gradually instead of sudden drop as in full separation observed in Celgard polymer separators manufactured via crazing. Similar observations have been reported for other non-woven materials, such as polypropylene fiber mats [23, 24].

During the normal battery operation the separator is not expected to sustain significant deformations, apart from those coming from the strains developed in electrodes with electrochemical cycling and from the cell stack pressure inside the battery pack. All of the commercially available separators are expected to safely deform under such strains without failure. Under mechanical abuse scenarios the situation obviously changes creating multiaxial stress-strain state which may lead to battery failure, i.e. internal short circuit with possible subsequent thermal runaway. The failure location and severity of the short circuit however has large degree of variability, - the factor that was the main driving force behind the development of new test procedure by the Battery Association of Japan [25]. In this test procedure, a cell is disassembled and a small conductive metal particle (nickel) is placed between the electrodes. The cell is then carefully put back together and the internal short is induced by applying pressure on the sell until the particle punctures the separator. The test was slightly modified by Celgard LLC [26]: instead of shorting the cell via nickel particle placed between electrodes, the small hole was punched in separator and the short was induced via direct contact between the electrodes under externally applied pressure. In this scenario, the area of the she contact is presumably known, which allows relating it with the severity of the short circuit.

While the experiments with controlled short circuit provided understanding on subsequent thermal behavior of cells up to thermal runaway, the mechanism of the separator failure is still rather poorly understood and is obviously dependent on the loading mode. An impact onto the electric vehicle $(\mathrm{EV})$ battery pack, such as occurring as a result of collision with road debris, can be approximated by indenting the pack with a hemispherical rigid object. In this scenario, very large deformations develop, including significant tensile strains which ultimately lead to the failure of separator. Such failure has been numerically simulated using homogenized material to represent battery cell [27]. It was shown later however, that resolving the electrodes and separator in the battery model leads to better predictive capability of the simulation [28]. Ultimately, it is the area where separator fails under the loading that determines the contact between the positive and negative electrodes and thus the amount of current available for rapid discharge. In this regard, the failure mode in which the separator fails into multiple thin strips under critical tensile strain will probably result in the largest contact area between the electrodes.

It should be noted, based on the reported results that the polymer separators possess remarkable strength in machine direction. This observation may challenge the general view that the separator does not play a role in overall battery strength and load bearing capability. When 
compared to the stress-strain response of electrodes [29] it can be noted that the tensile stress at fracture of an electrode is about 10 to 20 times smaller than that of a polymer separator loaded in machine direction (Figs. 5, 6). In transverse direction, the strength of a polymer separator approximately matches that of an electrode. The above indicates that battery separator is not only a passive membrane separating positive and negative electrodes but can also be a structural component in a battery. As such there has been research demonstrated towards enhancement of the battery strength through modifying separator properties. The approach generally consists of coating commercially available oxide particles $\left(\mathrm{SiO}_{2}\right.$ and $\left.\mathrm{Al}_{2} \mathrm{O}_{3}\right)$ onto the separator using polymer binder. These efforts mainly target thermal stability of separators [30-33]; however benefits to the mechanical properties have also been reported [33]. It is reasonable to expect the separator blended with ceramic particles to have higher stiffness in compression due to contact between the particles. However increase in tensile strength was also observed [33] which is critical for the safety of batteries.

The results reported thus far clearly show significant difference in strength, hardening, strain rate sensitivity and failure mechanism among the three commercially available separators tested in this investigation. It should be mentioned that the targets specifying separator mechanical properties are largely based on prevention of separator failure during the cell manufacturing [1]. The major requirement is therefore high tensile strength in machine direction since this is the direction of electrode stack winding. In addition to strength, elongation in MD and corresponding shrinkage in TD should be minimal (low Poisson's ratio) so that the edges of the electrodes would not be exposed and come into direct contact. A $0.2 \%$ offset yield stress of 6.9 $\mathrm{MPa}$ and higher in MD is described as acceptable criterion for most winding machines [1] and all three separators considered here satisfy this requirement. However, targeting only one parameter of mechanical behavior may not be sufficient, since as the results here show, while all separators pass the winding strength check, their other mechanical properties are quite different, which may have serious implications in case of a battery deformation during for instance vehicle crash. In case of multiaxial loading scenarios, strength in other directions becomes important.

\section{Conclusions}

Mechanical behavior of three types of commercial Li-ion battery separators (Celgard 2325, Celgard PP2075, and DreamWeaver Gold 40) was investigated in tension under a range of strain rates. The strain distribution was monitored by digital image correlation technique. The following can be concluded. Celgard separators show strong anisotropy and display significant rate sensitivity. Celgard 2325 exhibits cold drawing features in both directions, although with an order of magnitude difference in stress level; at the same time Celgard PP2075 behaves rubberlike in MD and displays cold drawing without work hardening in TD. The failure mode of Celgard PP2075 in MD significantly differs from that of Celgard 2325 in the same direction. The Celgard PP2075 separator fails by separating into multiple strips, while Celgard 2325 shows a clear tensile failure line perpendicular to the loading direction. Both of Celgard separators show non-uniform distribution of strain when stretched in transverse direction, - evidence of necking due to crazing. The DreamWeaver Gold 40 non-woven separator did not reveal any strain rate 
sensitivity or anisotropy of mechanical properties. Out of all three separators it showed the lowest elongation and failure strain.

\section{Acknowledgement}

This research at Oak Ridge National Laboratory, managed by UT-Battelle, LLC, for the U.S. Department of Energy under contract DE-AC05-00OR22725, was sponsored by the Vehicle Technologies Program for the Office of Energy Efficiency and Renewable Energy. Rick R. Lowden is acknowledged for his assistance and expertise in setting up the experiments.

\section{References}

[1] Baldwin, R.S., Bennett, W.R., Wong, E.K., Lewton, M. R., Harris, M.K., Battery separator characterization and evaluation procedures for NASA's advanced lithium-ion batteries, NASA/TM - $2010-216099$.

[2] ASTM D4830/D4830M - 98(2014) Standard test methods for characterizing thermoplastic fabrics used in roofing and waterproofing, ASTM International.

[3] Zhang, S.S., A review on the separators of liquid electrolyte Li-ion batteries, J Power Sources 164 (2007), 351-364.

[4] Lai, W.J., Ali, M., Pan, J., Mechanical behavior of representative volume elements of lithium-ion battery modules under various loading conditions." J Power Sources 248 (2014), 789-808.

[5] Sahraei, E., R. Hill, Wierzbicki, T., Calibration and finite element simulation of pouch lithium-ion batteries for mechanical integrity, J Power Sources,201 (2012): 307-321

[6] Cannarella, J., Arnold, C.B., Ion transport restriction in mechanically strained separator membranes, J Power Sources 226 (2013), 149-155.

[7] Venugopal, G., Moore, J., Howard, J., Pendalwar, S., Characterization of microporous separators for lithium-ion batteries, J Power Sources 77 (1999), 34-41.

[8] Sheidaei, A., Xiao, XR., Huang, XS, Hitt, J., Mechanical behavior of a battery separator in electrolyte solutions, J Power Sources 196(20) (2011), 789-808.

[9] Avdeev, I., Martinsen, M., Francis, A., Rate- and temperature-dependent material behavior of a multilayer polymer battery separator, J Mater Eng Performance 23 (2014), 315-325.

[10] Love, C.T., Thermomechanical analysis and durability of commercial micro-porous polymer Li-ion battery separators, J Power Sources 196 (2011), 2905-2912.

[11] Chen, J., Yan, Y., Sun, T., Qi, Y., Li, X., Deformation and fracture behaviors of microporous polymer separators for lithium ion batteries, RSC Adv, 4 (2014), 1490414914. 
[12] Cannarella, J., Liu, X., Leng, C.Z., Sinko, P.D., Gor, G.Y., Arnold, C.B., Mechanical properties of a battery separator under compression and tension, J Electrochem Soc 161(11) (2014), F3117-F3122.

[13] Gor, G.Y., Cannarella, J., Prevost, J.H., Arnold, C.B., A model for the behavior of battery separators in compression at different strain/charge rates, J Electrochem Soc, 161(11) (2014), F3065-F3071.

[14] Sugimoto, M., Ishikawa, M., Hatada, K., Toughness of polypropylene, Polymer 36(19) (1995), 3675-3682.

[15] Dasari, A., Rohrmann, J., Misra, R.D.K., Microstructural evolution during tensile deformation of polypropylenes, Mater Sci Engng A351 (2003), 200-213.

[16] Karger-Kocsis, J., Benevolenski, O.I., Moskala, E.J., Toward understanding the stress oscillation phenomenon in polymers due to tensile impact loading, J Mater Sci 36 (2001), 3365-3371.

[17] Ebener, H., Pleuger, B., Petermann, J., Stress and strain oscillations in syndiotactic Polypropylene and Poly(ethyleneterephthalate), J Appl Polymer Sci 71 (1999), 813-817.

[18] Toda, A., Tomita, C., Hikosaka, M., Hibino, Y., Miyaji, H., Nonomura, C., Suzuki, T., Ishihara, H., Thermo-mechanical coupling and self-excited oscillation in the neck propagation of PET films, Polymer 43 (2002), 947-951.

[19] Koenen, J.A., Observation of the heat exchange during deformation using an infra-red camera, Polymer 33(22) (1992), 4732-4736.

[20] Garcia Gutierrez, M.C., Karger-Kocsis, J., Riekel, C., Stress oscillation-induced modulated phase transformation and yielding in syndiotactic polypropylene, Chem Phys Lett 398 (2004), 6-10.

[21] Zhou, Y., Mallick, P.K., Effects of temperature and strain rate on the tensile behavior on unfilled and talc-filled polypropylene. Part I: Experiments, Polymer Eng Science 42(12) (2002), 2449-2460.

[22] Zhou, Y., Mallick, P.K., Effects of temperature and strain rate on the tensile behavior on unfilled and talc-filled polypropylene. Part II: Constitutive equation, Polymer Eng Science 42(12) (2002), 2461-2470.

[23] Jubera, R., Ridruejo, A., Gonzalez, C., Llorca, J., Mechanical behavior and deformation micromechanisms of polypropylene nonwoven fabrics as a function of temperature and strain rate, Mechanics of Materials, 74 (2014), 14-25.

[24] Ridruejo, A., Gonzalez, C., Liorca, J., Failure locus of polypropylene nonwoven fabrics under in-plane biaxial deformation, Recent Advances in Micromechanics of Materials, 340 (4-5) (2012), 307-319. 
[25] "1625-2008 - IEEE Standard for Rechargeable Batteries for Multi-Cell Mobile Computing Devices." IEEE SA -. Web. 20 Jan. 2015. http://standards.ieee.org/findstds/standard/16252008.html.

[26] Santhanagopalan, S., Ramadass, P., Zhang, Z., Analysis of internal short-circuit in a lithium ion cell, J Power Sources 194(1) (2009), 550-557.

[27] Sahraei, E., Hill, R., Wierzbicki, T., Calibration and finite element simulation of pouch lithium-ion batteries for mechanical integrity, J Power Sources 201 (2012), 307-321.

[28] Kumar, A., Kalnaus, S., Simunovic, S., Gorti, S., Allu, S., Turner, J.A., Communication Indentation of Li-ion pouch cell : Effect of material homogenization on prediction of internal short circuit, J Electrochem Soc., 163(10) (2016), A2494-A2496.

[29] Lai, W.-J., Ali, M.Y., Pan, J., Mechanical behavior of representative volume elements of lithium-ion battery modules under various loading conditions, J Power Sources, 248 (2014), 789-808.

[30] Choi, J.-A., Kim, S.H., Kim, D.-W., Enhancement of thermal stability and cycling performance in lithium-ion cells through the use of ceramic-coated separators, J Power Sources 195(18) (2010), 6192-6196.

[31] Jeong, H.-S., Kim, D.-W., Jeong, Y.U., Lee, S-Y., Effect of phase inversion on microporous structure development of A12O3/poly(vinylidene fluoridehexafluoropropylene)-based ceramic composite separators for lithium-ion batteries, J Power Sources 195(18) (2010), 6116-6121.

[32] Shin, W.-K., Kim, D.-W., High performance ceramic-coated separators prepared with lithium ion-containing $\mathrm{SiO}_{2}$ particles for lithium-ion batteries, J Power Sources 226 (2013), 54-60.

[33] Fu, D., Luan, B., Argue, S., Bureau, M.N., Davidson, I.J., Nano SiO2 particle formation and deposition on polypropylene separators for lithium-ion batteries, J Power Sources 206 (2012), 325-333. 